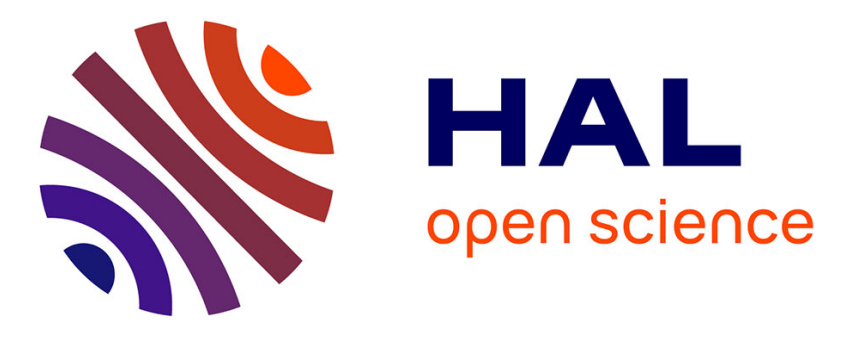

\title{
Land change modeling: moving beyond projections
}

Martin Paegelow, Maria T. Camacho Olmedo, Jean-François Mas, Thomas

Houet, Gilmore Robert Pontius

\section{To cite this version:}

Martin Paegelow, Maria T. Camacho Olmedo, Jean-François Mas, Thomas Houet, Gilmore Robert Pontius. Land change modeling: moving beyond projections. International Journal of Geographical Information Science, 2013, 27 (9), pp.1691-1695. 10.1080/13658816.2013.819104 . halshs-00905367

\section{HAL Id: halshs-00905367 https://shs.hal.science/halshs-00905367}

Submitted on 19 Dec 2013

HAL is a multi-disciplinary open access archive for the deposit and dissemination of scientific research documents, whether they are published or not. The documents may come from teaching and research institutions in France or abroad, or from public or private research centers.
L'archive ouverte pluridisciplinaire HAL, est destinée au dépôt et à la diffusion de documents scientifiques de niveau recherche, publiés ou non, émanant des établissements d'enseignement et de recherche français ou étrangers, des laboratoires publics ou privés. 


\title{
Editorial Special Issue
}

\section{Land Change Modeling: moving beyond projections}

\author{
Paegelow M., Camacho Olmedo M. T., Mas J.-F., Houet T., Pontius G. R.
}

During the last decades, there has been an increasing interest from the academic and policy communities to monitor and model changes of the earth surface. Modeling environmental dynamics helps to understand changes that are taking place currently and to anticipate future evolutions. Prospective simulation supports decision-making for environmental management and land planning. This special issue is dedicated to advances in land change modeling. Land Use / Land Cover Change, also called LUCC, is certainly a prominent interface between natural and social dynamics because anthropogenic LUCC has a profound impact on Earth. LUCC impacts a large amount of highly relevant topics such as resource exploitation, climate change, biodiversity loss, etc. Land change modeling can provide transparent, efficient and sustainable decision support to these current and rising environmental problems. The International Journal of Geographic Information Science (IJGIS) has contributed to progress in LUCC research by publishing excellent papers on land change modeling and special issues such as "Spatial agent-based modeling" (Vol 20, issue 9, 2006) and "Spatial modeling to explore land use dynamics" (Vol 19, issue 2, 2005). IJGIS has also published special issues concerning related topics, such as "Spatial Ecology" (Vol 25, issue 3, 2011, Vol 26, issue 11, 2012) and "Object-based landscape analysis" (Vol 25, issue 6, 2011).

Where do we come from? Land change modeling means the simulation of the behavior of an environmental and social system in space and through time in a manner that relates to land change. This research involves many challenges such as comprehension of complex environmental processes and decision support for environmental management \& land planning. Simulation modeling of 'what would happen if' has become a routine analytic approach in many domains such as risk prevention, environmental impact studies and land planning. Therefore scientists use geomatics and, especially, Geographic Information Systems (GIS) that deal with spatio-temporal data. At first, GIS was able only to process spatial data, such as performing Boolean combinations of different layers or querying attributes. As recently as the 1990s, geomatics software did not usually inject time into analytic tools. During the last two decades, GIS software has been enhanced with new components to process the time dimension of data in order to analyze past trends and to model the future. During the last two decades, the methodological approaches involved in land change modeling were profoundly enhanced and became popular in human and social sciences. Classical stochastic methods became complemented by cellular automaton, multi-agent systems and artificial intelligence. The first books on land change modeling were published in the 1990s. By the end of the 1990s, first overviews about modeling approaches and underlying concepts were published.

Where are we now? Most recently, substantial work has been done concerning how to handle time. Time is involved in both prospective and retrospective simulation. Temporal extents can range from short to long, while temporal resolutions that can range from fine to coarse. Decisions concerning temporal scales are a common challenge in literature. The notions of temporality, granularity, spatiotemporal continuum, stationarity, instability and intensity change analysis are still some of the points of contention in the question of how to integrate time in modeling. Similar conceptual challenges exist with respect to spatial scales and categorical scales. Land change modeling responds to a growing social demand because land change can be an indicator of irreversible processes.

Yesterday's stochastic methods are complimented today by a wide range of techniques including artificial intelligence, multi-agent systems, cellular automaton, fuzzy logic, uncertainty analysis and methods to address missing data. In parallel, the objectives of land change modeling have become more diversified. Models can help to understand complex environmental systems, to validate extrapolations and to design alternative future scenarios. Retrospective simulation can 
extrapolate historic trends to measure predictive power over a more recent validation interval. This approach can be helpful to appreciate the uncertainty in outputs as models project into an unknown future. However, retrospective simulation has limitations, because there are many various ways to measure predictive power during a validation interval. Moreover, some models are designed to simulate processes and decisions that have no historical precedent, in which case validation according to past patterns makes little sense. The objectives of land change modeling extend beyond extrapolation of past trends. A knowledge objective is to increase understanding of temporal processes. A methodological objective is to define the degree of a model's generalization. An operational objective is to examine the likely impacts of various possible management decisions.

Moving beyond projections: What is coming on? Non-linear behavior of LUCC is one of the challenges that land change modeling must face. For example, future LUCC will occur in a global climate with which modern humans have had no experience. Therefore LUCC models must consider non-stationary spatial systems with changing driving forces and processes. Another challenge is to design integrated modeling systems to deal with uncertainty concerning future trends that have no historical precedence. A constant challenge is to create models that relate directly to land planning and environmental management. Therefore we need more studies concerning generalization and standards for putting these models into practice.

In order to progress, the next generation of land change models must make methodological and technical advances. From a predictive point of view, we need clearer methods to assess accuracy concerning landscape dynamics, including their spatial structure. From a scenario-based point of view, we need tools to assess the plausibility of changes and the diversity of possible land changes. Furthermore, models must address data uncertainty, data errors and lack of data. An additional challenge is the validation of simulations and scenarios that combine measures of disagreement in quantity, allocation and pattern. To offer sustainable solutions, land change modeling has to be transparent and include participatory elements.

This special issue is a selection of 6 articles among 30 submitted papers. The first two articles illustrate progress in the field of characterizing data, by measuring the stationarity in a temporal sequence of maps and by considering whether errors in the maps can account for observed patterns. The third article is an example of land change modeling beyond the academic sphere, as it shows how modeling has becomes integrated in land planning and environmental management. The fourth article develops sensitivity analysis to detect uncertainty in simulated change in the framework of agent based modeling, while the fifth article shows model sensitivity depending on how the model is applied. The sixth article pays attention to land change drivers and how these drivers influence the effectiveness of LUCC models. We summarize each article below.

Runfola and Pontius offer a method to measure the stability, i.e. stationarity, of patterns in a temporal sequence of maps that show land categories. This is important because models frequently calibrate parameters based on maps of historic changes among categories. Then such models often extrapolate the historic trends to create a business-as-usual scenario. However, if the historic trends have not been stable, then business has not been usual, so it makes no sense to construct a businessas-usual scenario. Runfola and Pontius introduce a novel matrix, called the Flow matrix, to measure historic stability. The Flow matrix is an important alternative to the popular Markov matrix. Runfola and Pontius use the Flow matrix to compare the stability of land changes for numerous sites. Researchers can use their method to understand historic patterns, which is advisable before trying to simulate those patterns into the future.

Aldwaik and Pontius also measure the patterns in a temporal sequence of maps that show land categories. They use Intensity Analysis, which is an established mathematical framework that analyzes a transition matrix for each time interval for consecutive intervals. Their paper presents a method to measure whether errors in the maps can account for deviations from uniform land changes. They designed their method to address a common type of situation, where researchers do not know the sizes and types of error in the maps. This situation is common because maps of historic 
land cover are plentiful due in part to the prevalence of satellite images. But many historic maps lack information concerning accuracy and it is impossible to go back in time to obtain the ground reference information that would be necessary to assess accuracy. If researchers have the maps, then they can measure the differences in the maps, but they will never know whether errors in the maps are the reason for the differences. The method of Aldwaik and Pontius computes the minimum hypothetical error that could account for observed differences in the context of Intensity Analysis. Then those minimum hypothetical errors can be compared to the errors that the researcher suspects are in the maps, based on the researcher's experience with maps.

Mubareka et al present a modeling chain, based on Land Use Modeling Platform (LUMP) developed by the Joint Research Centre of the European Union, in order to assess the impacts of natural water retention measures on Europe's green infrastructure. Mubareka et al's article is highly relevant for policy driven questions. It is notable because the reference space is as large as the European Union, and the authors use data concerning a wide variety of economic and social drivers. Mubareka et al's contribution illustrates an important challenge for land change modeling, i.e. to integrate modeling with land planning.

Ligmann-Zielinska investigates the spatial dimension of sensitivity analysis in land use change modeling. She employs a model-independent approach to sensitivity analysis that decomposes the variance of model output to compute sensitivity indices of model inputs. Using an agent-based model of residential development, she proposes two different procedures of analyzing model sensitivity. First, she uses the maps indirectly by applying variance decomposition to output land change maps summarized into scalar pattern metrics. Second, she uses the maps as a direct input to variance decomposition, and calculates the sensitivity indices for every spatial unit within the area of study. The proposed method provides the necessary means of investigating the spatial dependence of land use change, its accompanying uncertainty, and the heterogeneity of the essential determinants of the simulated land use maps. The reported computational experiments demonstrate that the decision whether to select the spatially independent or the spatially dependent approach should be considered carefully, because the methods may lead to a different prioritization and reduction of model input parameters.

Kim et al. reveal the general pattern of variation in land change simulation outcomes due to changes in four sensitivity experiments concerning spatial scale, temporal scale, probability distribution and degree of the influence of stochastic factors. This is accomplished using a hypothetical model that reflects the key logic and common working principles of various cell-based dynamic land use change modeling environments. The approach has the advantage that the influence of the simulation settings can be controlled. As land use modelers are dealing with uncertainties, this work helps to understand better why models generate dissimilar or similar simulation outcomes under different scenarios, depending on whether the difference is attributable to a change in key parameters across scenarios or, to the detailed application settings combined with demand growth trajectories. The main contribution of this work is to enhance the understanding of complex model behaviors in and across various applications. The approach can support more informed model implementation.

Kolb et al. analyze LUCC drivers, giving in-depth insights of their varying influence in LUCC processes during two periods, i.e. 1993-2002 and 2002-2007. They consider two different methods to calibrate LUCC models: Per transition change probability maps produced by the Weights of evidence (WoE) and Per land cover category suitability maps obtained by Logistic Regression Modeling (LRM). They show that the change probability obtained by WoE is more accurate at predicting change, whereas the suitability map produced by LRM is better at predicting persistence. This paper highlights how LUCC drivers can evolve over time, whereas WoE and LRM are appropriate to project land change when the drivers are stationary, thus the techniques have some limitations for projecting various futures. One important lesson is that modelers must consider path-dependence and possible disruptions of future LUCC when designing scenarios. 
The editors are grateful for receiving support from MINECO I+D+i'. We thank all authors who submitted manuscripts. The editors and the authors are indebted to the reviewers who spent time and effort to offer constructive feedback on submitted manuscripts. Reviewers' expertise contributed substantially to improve this special issue. Reviewers were: Arnon Accad, Franciso Aguilera Benavente, Claudia Almeida, Annalisa Appice, Richard Arnott, Claudia Baranzelli, José Barredo, Nicolas Becu, Andrew Bell, Itzhak Benenson, David Bennet, Roger Bivand, Gerardo Boco Verdinelli, Daniel Brown, Marie Castellazzi, Leonardo Chapa-Vargas, Hao Chen, Claude Collet, Helen Couclelis, Andrew Crooks, Laurent Durieux, Francisco Escobar, Ghislain Geniaux, Thomas Glade, Candan Gokceoglu, Montserrat Gomez-Delgado, Joon Heo, Jeffrey Hepinstall, Irmela Herzog, Barbara Hofer, Bo Huang, John Harrington, Thierry Joliveau, Stéphane Joost, Eric Koomen, Xiaohui Liu, Xiaoping Liu, Eva Malinverni, Steven Manson, Joseph Messina, James Millington, Antonio Monteiro, Fraser Morgan, Irmgard Niemeyer, Wenseslao Plata Rocha, Carla Roca Gomes, Daniel Runfola, Mehdi Saqalli, David Sheeren, Britaldo Soares-Filho, Terry Sohl, Stephen Stehman, Marius Theriault, JeanClaude Thiel, Tom Veldkamp, Peter Verburg, Michael Widener and Ninqchuan Xiao.

'Ministerio de Economía y Competitividad MINECO, Spain. Project I+D+i: "Simulaciones geomáticas para modelizar dinámicas ambientales. Avances metodológicos y temáticos". 2009-2012. BIA2008-00681 